\title{
Methicillin-resistant Staphylococcus epidermidis isolation from the vitrectomy specimen four hours after initial treatment with vancomycin and ceftazidime
}

\author{
This article was published in the following Dove Press journal: \\ Clinical Ophthalmology \\ 23 February 2010 \\ Number of times this article has been viewed
}

\author{
Golnaz Javey' \\ Stephen G Schwartz \\ Andrew A Moshfeghi \\ Sanjay Asrani ${ }^{3}$ \\ Harry W Flynn J $r^{2}$ \\ 'Department of Ophthalmology, \\ Cullen Eye Institute, Baylor College \\ of Medicine, Houston, TX, USA; \\ ${ }^{2}$ Department of Ophthalmology, \\ Bascom Palmer Eye Institute, \\ University of Miami Miller School \\ of Medicine, Miami, FL, USA; \\ ${ }^{3}$ Department of Ophthalmology, Duke \\ University Medical Center, Durham, \\ NC, USA
}

\begin{abstract}
A patient presented with acute-onset, postoperative endophthalmitis and visual acuity of light perception. Because of a time delay in arranging a pars plana vitrectomy (PPV), the patient was treated with a prompt vitreous tap for culture an injection of vancomycin and ceftazidime. Four hours later, the PPV was performed and additional antibiotics were injected. The cultures from both the initial needle tap and the subsequent PPV isolated methicillinresistant Staphylococcus epidermidis sensitive to vancomycin, but resistant to fourth-generation fluoroquinolones. The patient eventually recovered a visual acuity of 20/80 before developing retinal detachment. This case illustrates the time lag necessary to sterilize the vitreous cavity, and suggests a possible two-step staged treatment strategy for situations in which access to PPV equipment and support staff may be limited.
\end{abstract}

Keywords: endophthalmitis, pars plana vitrectomy, tap and inject

\section{Introduction}

Acute-onset, postoperative endophthalmitis is an uncommon but potentially severe complication of cataract surgery. ${ }^{1}$ Successful treatment of endophthalmitis requires accurate microbiologic diagnosis and prompt treatment with effective antimicrobial agents. $^{2}$ The Endophthalmitis Vitrectomy Study (EVS) reported that, for patients with acute-onset, postoperative endophthalmitis and presenting visual acuity of light perception (LP), initial treatment with pars plana vitrectomy (PPV) and intravitreal antibiotics was associated with more favorable visual outcomes than was treatment with vitreous tap and intravitreal antibiotics. ${ }^{3}$ In the EVS, PPV was performed within six hours of the initial examination, ${ }^{3}$ but this may not always be feasible in clinical practice. One alternative approach is to perform prompt vitreous tap and injection of intravitreal antibiotics, followed by PPV as soon as practicable. We present a case of acute-onset, postoperative endophthalmitis managed in this fashion.

\section{Case report}

An 86-year-old male presented five days after uncomplicated phacoemulsification and posterior chamber intraocular lens implantation, with a 24-hour history of progressive visual loss and redness in the operated (left) eye. Past medical history was significant for hypertension, dyslipidemia, sleep apnea, and essential tremor. Medications included lisinopril, atorvastatin, amlodipine, and propanolol. There were no known drug allergies. On examination, visual acuity was LP. Slit-lamp examination demonstrated
Correspondence: Stephen G Schwartz Bascom Palmer Eye Institute at Naples, 311 9th Street North, \#100, Naples, FL 34102 , USA

Tel +l 2396593937

Fax + I 2396593982

Email sschwartz2@med.miami.edu 


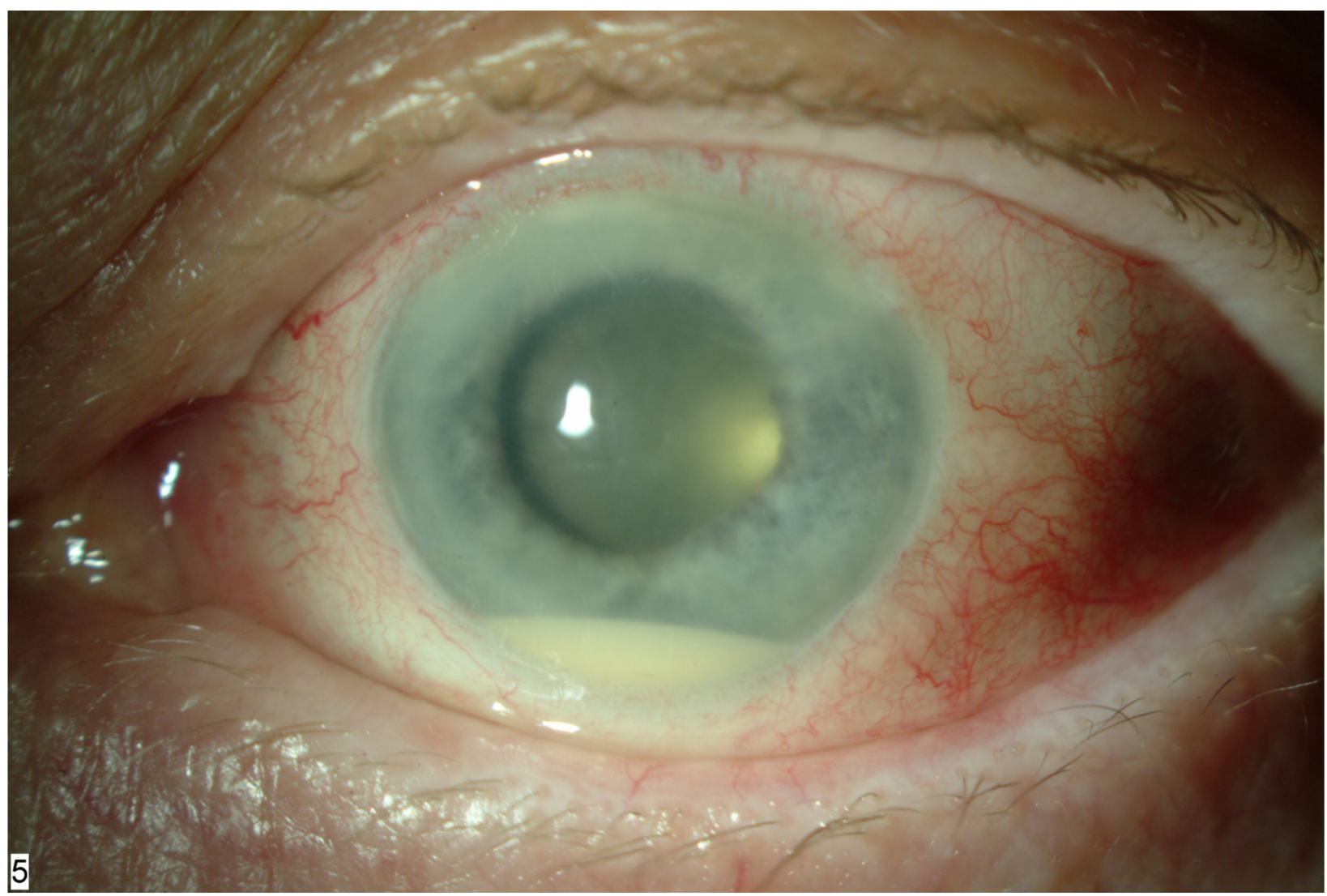

Figure I Slit lamp photograph, left eye, demonstrated layered hypopyon and fibrin formation in the anterior chamber.

conjunctival injection, corneal edema, and hypopyon (Figure 1). Dilated fundus examination showed dense vitreous opacification. B-scan echography demonstrated vitreous opacification, but no retinal detachment (Figure 2).

The patient had presented to a satellite clinic in a suburban location. Access to an operating room with PPV equipment and appropriate support staff would not be possible for at least several hours. After a full discussion with the patient, it was decided to perform prompt vitreous tap and injection of intravitreal antibiotics in an attempt to stabilize the eye, followed by PPV as soon as the operating room, vitrectomy equipment, and the appropriate surgical team could be secured. Aqueous and vitreous cultures were obtained by needle tap, and the eye was treated with intravitreal injection of vancomycin $1 \mathrm{mg} / 0.1 \mathrm{~mL}$ and ceftazidime $2.25 \mathrm{mg} / 0.1 \mathrm{~mL}$. Systemic antibiotics were not administered.

Approximately four hours after the vitreous tap and invitreal injection of antibiotics, an operating room became available and a 23-gauge PPV was performed. Using the vitreous cutter, undiluted vitreous was obtained prior to initiating infusion of balanced salt solution. Intraoperative findings included dense vitreous opacification and a superior retinal break located posteriorly near the equator, which was treated with endolaser photocoagulation. There did not appear to be a posterior vitreous detachment (PVD) at the time of surgery, and no attempt was made to induce a PVD surgically. Vancomycin $1 \mathrm{mg} / 0.1 \mathrm{~mL}$ and ceftazidime $2.25 \mathrm{mg} / 0.1 \mathrm{~mL}$ were re-injected into the vitreous cavity at the conclusion of

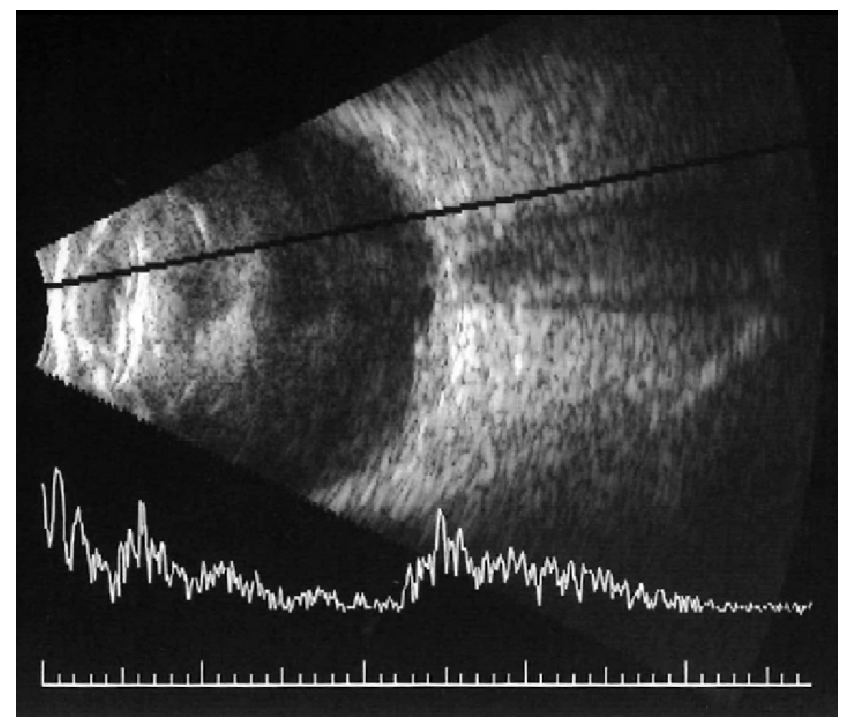

Figure 2 B-scan echography, left eye, demonstrated vitreous opacities but no retinal detachment. 
surgery. Fluid-air exchange was not performed. Postoperatively, the patient was treated with topical moxifloxacin and prednisolone acetate 1\%. Again, systemic antibiotics were not administered.

The vitreous cultures from both the initial needle tap and the subsequent PPV isolated methicillin-resistant Staphylococcus epidermidis sensitive to vancomycin. The patient demonstrated clinical improvement and one month later, visual acuity improved to 20/80. Unfortunately, the patient subsequently developed retinal detachment. A repeat PPV was performed, at which time epiretinal membranes, but no additional breaks, were identified. The patient was treated with PPV, membrane peeling, inferior retinectomy, and placement of silicone oil. At three months following presentation, visual acuity was 20/200 with silicone oil tamponade.

\section{Discussion}

In the EVS, $70 \%$ of microbiological isolates contained coagulase-negative micrococci. ${ }^{3}$ In this case, persistent coagulase-negative staphylococci were isolated from the PPV culture four hours following treatment with vancomycin and ceftazidime. Antibiotic kill rates are affected by drug concentration as well as contact time with the microorganisms. Vancomycin inhibits bacterial cell wall synthesis by interfering with peptidoglycan synthesis. Vancomycin is initially bacteriostatic and then becomes bactericidal after about eight hours. ${ }^{4}$ The serum killing curves of vancomycin against $S$. aureus demonstrate a lag phase prior to a rapid killing phase. This case demonstrates that sterilization of the vitreous cavity is by no means immediate. A single intravitreal injection may not sterilize the vitreous cavity even after several days. In the EVS, of the 420 patients enrolled, $33(8 \%)$ were re-cultured within the first week because of clinical signs of worsening infection or inflammation, and of these 33 patients, 14 (42\%) were persistently culturepositive. Persistent positive cultures were more common in patients randomized to initial needle tap (71\%) than those randomized to initial PPV (13\%), and this difference was statistically significant $(P=0.001) .^{5}$

Although the EVS reported an association between PPV and more favorable visual outcomes, PPV is usually performed in an operating room with specialized equipment and support staff. In some circumstances, it may not be feasible to perform PPV within a sufficiently timely fashion, and a staged two-step treatment potentially offers the benefits of faster initiation of antimicrobial therapy. In the EVS, among the subset of patients with LP vision treated with PPV, 11\% achieved visual acuity of $20 / 40$ or better, $30 \%$ achieved visual acuity of $20 / 100$ or better, and $47 \%$ developed severe visual loss, defined as visual acuity of worse than 5/200. Therefore, this patient, treated with a staged two-step treatment, achieved a visual outcome consistent with that reported in the EVS.

This patient was not treated with systemic antibiotics. The EVS reported no significant difference in outcomes with or without the use of systemic amikacin and ceftazidime. ${ }^{3}$ Newer fourth-generation fluoroquinolones, such as gatifloxacin ${ }^{6}$ and moxifloxacin, ${ }^{7}$ achieve potentially therapeutic intraocular drug levels in the noninflamed eye. Although their benefit in the clinical management of acute-onset, postoperative endophthalmitis remains unproven, systemic fluoroquinolones may be considered in cases similar to this one. Systemic gatifloxacin is no longer commercially available in the United States due to associated risks of hyperglycemia and hypoglycemia. ${ }^{8}$

During the initial PPV, a superior retinal break was identified. This break may have been caused by the endophthalmitis, by the initial vitreous tap and inject, or by the PPV. The eye initially improved to a visual acuity of 20/80 but then developed retinal detachment, which required further surgery. It is possible that the staged two-step treatment may have increased the risk of retinal detachment, although it is also possible that a staged two-step treatment may have improved the final outcome.

In the setting of acute-onset, postoperative endophthalmitis with visual acuity of LP and no prompt access to an operating room, a staged two-step treatment (tap and inject followed by PPV) may be a reasonable treatment option. It is obviously very difficult to draw definitive conclusions from single cases, and further clinical experience is necessary to validate this report.

\section{Disclosures}

Partially supported by NIH center grant P30-EY014801, by an unrestricted grant to the University of Miami from Research to Prevent Blindness, New York, NY, and by The Palm Beach Community Trust Fund, Palm Beach, FL. The authors have no proprietary interests in the subject of this manuscript.

\section{References}

1. Miller DM, Vedula AS, Flynn HW Jr, et al. Endophthalmitis caused by staphylococcus epidermidis: in vitro antibiotic susceptibilities and clinical outcomes. Ophthalmic Surg Lasers Imaging. 2007;38:446-451.

2. Endophthalmitis Vitrectomy Study Group. Microbiologic factors and visual outcomes in the Endophthalmitis Vitrectomy Study. Am J Ophthalmol. 1996;122:830-846. 
3. Endophthalmitis Vitrectomy Study Group. Results of the Endophthalmitis Vitrectomy Study: a randomized trial of immediate vitrectomy and of intravenous antibiotics for the treatment of postoperative bacterial endophthalmitis. Arch Ophthalmol. 1995:113(12):1479-1496.

4. Libre P, Della-Latta P, Chin N. Intracameral antibiotic agents for endophthalmitis prophylaxis: a pharmacokinetic model. J Cataract Refract Surg. 2003;29:1791-1794.

5. Doft BH, Kelsey SF, Wisniewski SR. The EVS study group. Additional procedures after the initial vitrectomy or tap-biopsy in the endophthalmitis vitrectomy study. Ophthalmology. 1998;105:707-716.
6. Hariprasad SM, Mieler WF, Holz ER. Vitreous and aqueous penetration of orally administered gatifloxacin in humans. Arch Ophthalmol. 2004; 121:345-350

7. Hariprasad SM, Shah GK, Mieler WF, et al. Vitreous and aqueous penetration of orally administered moxifloxacin in humans. Arch Ophthalmol. 2006;124:178-182.

8. Park-Wyllie LY, Juurlink DN, Kopp A, et al. Outpatient gatifloxacin and dysglycemia in older adults. $N$ Engl J Med. 2006;354:1352-1361.
Clinical Ophthalmology

\section{Publish your work in this journal}

Clinical Ophthalmology is an international, peer-reviewed journal covering all subspecialties within ophthalmology. Key topics include: Optometry; Visual science; Pharmacology and drug therapy in eye diseases; Basic Sciences; Primary and Secondary eye care; Patient Safety and Quality of Care Improvements. This journal is indexed on

\section{Dovepress}

PubMed Central and CAS, and is the official journal of The Society of Clinical Ophthalmology (SCO). The manuscript management system is completely online and includes a very quick and fair peer-review system, which is all easy to use. Visit http://www.dovepress.com/ testimonials.php to read real quotes from published authors. 